\title{
Entire-Domain Basis Function with Segmented Edge Condition Applied for Scattering Structures
}

\author{
Edson R. Schlosser ${ }^{1}$ (D), Marcos V. T. Heckler ${ }^{1}$ (D), José R. Bergmann ${ }^{2}$ (D) \\ ${ }^{1}$ Laboratory of Electromagnetics, Microwaves and Antennas - LEMA, Federal University of Pampa \\ (UNIPAMPA),Alegrete 97546-550,Brazil,edsonschlosser@unipampa.edu.br, marcos.heckler@unipampa.edu.br \\ ${ }^{2}$ Centre for Telecommunications Studies - CETUC, Pontifical Catholic University of Rio de Janeiro (PUC-Rio), \\ Rio de Janeiro 22451-900, Brazil, bergmann@puc-rio.br
}

\begin{abstract}
This paper proposes the formulation of a sinusoidal basis function with a novel segmented edge condition to model the impulsive behavior of the surface electric current density at the edges of rectangular microstrip scatterers. In comparison to traditional basis functions, the one considered in this approach demands using very few modes to expand the induced current. The effectiveness of the proposed formulation is validated using the commercial electromagnetic simulator Ansys Designer. Good agreement between the results obtained with the proposed formulation and with the commercial software has been obtained.
\end{abstract}

Index Terms - printed antennas; scattered fields; method of moments; basis function.

\section{INTRODUCTION}

The interest on printed structures has increased due to the evolution of electromagnetic and mechanical properties of dielectric laminates. Materials with low losses in the microwave range have allowed the design with high efficiency of microstrip antenna arrays, reflectarrays (RAs), transmitarrays (TAs), polarizers, and frequency selective surfaces (FSS).

The electromagnetic analysis of printed structures can be realized using academic codes or commercial software, such as Ansys Designer, HFSS, FEKO and CST [1]-[3]. These solutions are based on numerical techniques, such as the method of moments (MoM), finite element method (FEM), finite integration technique (FIT) and finite-difference time-domain (FDTD) [4]-[6].

The efficiency and the accuracy of the MoM numerical scheme depends on the choice of the basis functions (BFs), where two classes of BFs are usually employed: subdomain function and entire-domain function [7]. Rooftop, pyramidal with rectangular base, pyramidal with triangular base, and Rao-WiltonGlisson (RWG) are examples of subdomain basis functions [8], while sinusoidal basis functions are traditional examples of entire-domain basis functions, defined on the complete structure [9].

For designs composed of rectangular printed elements, specific entire-domain basis functions have been applied to reduce the total number of functions needed to model the impulsive behavior of the induced electrical current density at the edges of the scatterers. Sinusoidal function with edge condition, Legendre (E-LegBF), and Chebyshev (E-CheBF) are examples of BFs with these features typically applied for the solution of scattering problems, as presented in [10]-[14]. Additionally, in [15] the application of BFs in reflectarray antennas composed of rectangular patches or dipoles is discussed and the frequency displacement of the phase and amplitude curves for several types of basis functions 
is also presented. The conclusion indicates that these basis functions show good agreement only for substrates with thickness greater than 0.04 wavelengths, and show poor convergence for extremely thin substrates. However, the design of reflectarray antennas using single layer structures composed of variable-size elements requires thin laminates to extend the range of the phase curves.

In this paper, we propose an entire-domain basis function with segmented edge condition to model the surface current density of rectangular patches with variable sizes, and, consequently, to determine the amplitude and phase curves of the element using very few expansion modes. In order to accurately describe the induced current on the patch, this type of basis function is segmented in different patch regions, so as to allow the modelling of the steep increase of the current density at the non-resonant edges of the patch. A segmentation factor allows the adjustment of function behavior for substrates with different thicknesses; hence, in contrast to [15], the proposed basis function can be easily applied for thin substrates with thickness lower than 0.04 wavelengths.

Here, the proposed basis function is employed with the method of moments (MoM) for the accurate computation of the scattered fields of reflectarrays. In Section 2, the formulations of the dyadic Green's function, the method of moments, and the basis function with segmented edge condition are presented. In section 3, the amplitude and phase curves for the scattered field of a single patch are presented. Additionally, the scattered electric fields by reflectarrays with few elements are validated using Ansys Designer. Section 4 presents the final remarks containing the advantages of the proposed basis function.

\section{THEORETICAL Formulation}

The geometry of a singly-layered rectangular microstrip scatterer illuminated by a plane wave is depictured in Fig. 1. Here, the electromagnetic analysis follows the steps presented in [16] where the scattered electric field in the spectral domain depends on the dyadic Green's function and the surface electric current density $\mathbf{J}^{(s)}$. The physical characteristics of the structure defines the dyadic Green's function, whilst the surface electric current density is unknown in the presence of a printed element on the substrate. The current distribution can be determined using appropriate numerical techniques. Here, the method of moments (MoM) is chosen and formulated to determine the scattered electric field produced by $\mathbf{J}^{(s)}$ that flows on the patch [17].

\section{A. Dyadic Green's function}

The structure presented in Fig. 1 is homogeneous, infinite, and can be represented by using an equivalent circuit model [16], where $\mathbf{E}^{(s)}$ is the scattered electric field and $\mathbf{E}^{(i n c)}$ is the electric field of the wave that impinges upon de scatterer. The relation between the scattered electric field and the surface current density that flows on the patch is described in the spectral domain by

$$
\tilde{\mathbf{E}}^{(s)}=j \eta_{0} \overline{\tilde{G}} \tilde{\mathbf{J}}^{(s)} \rightarrow\left[\begin{array}{c}
\tilde{E}_{y}^{(s)} \\
\tilde{E}_{x}^{(s)}
\end{array}\right]=j \eta_{0} \overline{\tilde{G}}\left[\begin{array}{c}
\tilde{J}_{y}^{(s)} \\
\tilde{J}_{x}^{(s)}
\end{array}\right],
$$

where $\eta_{0}$ is the intrinsic impedance of free space, $k_{0}$ is the propagation constant in free space, $d_{m}$ is the substrate thickness, $\varepsilon_{r_{\tau}}$ is the relative permittivity of $\tau$ region, and $\overline{\tilde{G}}$ is the dyadic Green's function, given by

$$
\overline{\tilde{G}}=\left[\begin{array}{cc}
\tilde{G}_{y y} & \tilde{G}_{y x} \\
\tilde{G}_{x y} & \tilde{G}_{x x}
\end{array}\right]=\left[\overline{\tilde{Y}}^{(a)}+\overline{\tilde{Y}}^{(b)}\right]^{-1},
$$

Brazilian Microwave and Optoelectronics Society-SBMO

Brazilian Society of Electromagnetism-SBMag received 30 Jan 2021; for review 18 Feb 2021; accepted 28 June 2021 (C) 2021 SBMO/SBMag (cc) BY

ISSN 2179-1074 


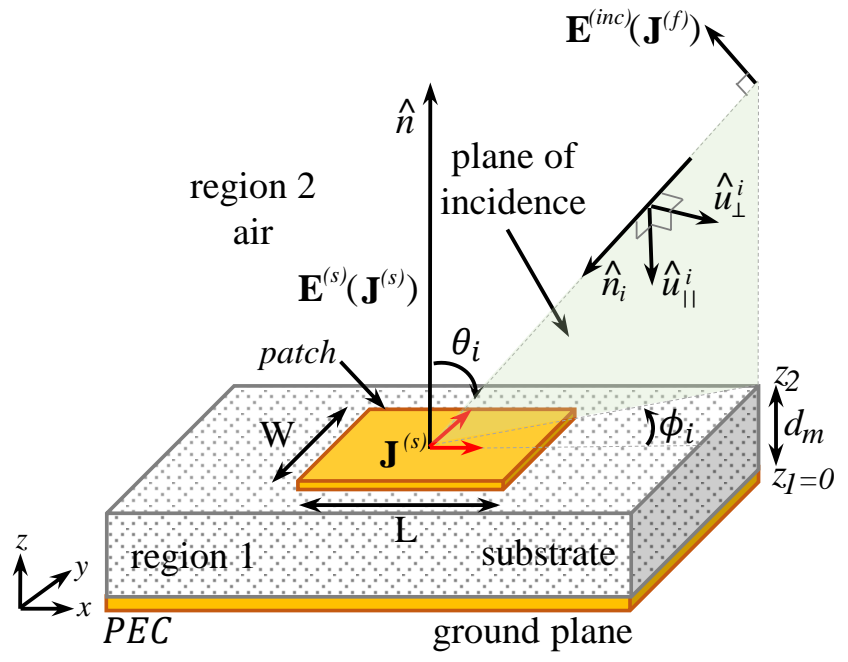

Fig. 1. Basic geometry of a singly-layered rectangular microstrip scatterer illuminated by a plane wave.

where $\overline{\tilde{Y}}^{(a)}$ and $\overline{\tilde{Y}}^{(b)}$ are the admittances seen looking out of the current source terminals (see circuit model shown in Fig. 2) and are expressed by

$$
\begin{gathered}
\overline{\tilde{Y}}^{(a)}=-\frac{1}{k_{0} k_{z_{2}}}\left[\begin{array}{cc}
\left(\varepsilon_{r_{2}} k_{0}^{2}-k_{x}^{2}\right) & k_{x} k_{y} \\
k_{x} k_{y} & \left(\varepsilon_{r_{2}} k_{0}^{2}-k_{y}^{2}\right)
\end{array}\right], \\
\overline{\tilde{Y}}^{(b)}=\overline{\tilde{B}} \overline{\tilde{Z}}^{-1}, \\
\overline{\tilde{B}}=\left[\begin{array}{cc}
\cosh \left(k_{z_{1}} d_{m}\right) & 0 \\
0 & \cosh \left(k_{z_{1}} d_{m}\right)
\end{array}\right], \\
\overline{\tilde{Z}}=\frac{\sinh \left(k_{z_{1}} d_{m}\right)}{\varepsilon_{r_{1}} k_{0} k_{z_{1}}}\left[\begin{array}{cc}
\left(k_{y}^{2}-\varepsilon_{r_{1}} k_{0}{ }^{2}\right) & k_{x} k_{y} \\
k_{x} k_{y} & \left(k_{x}^{2}-\varepsilon_{r_{1}} k_{0}{ }^{2}\right)
\end{array}\right],
\end{gathered}
$$

and

$$
k_{z_{\tau}}^{2}=k_{x}^{2}+k_{y}^{2}-\varepsilon_{r_{\tau}} k_{0}^{2},
$$

with $\tau=1$ for the substrate and $\tau=2$ for the air.

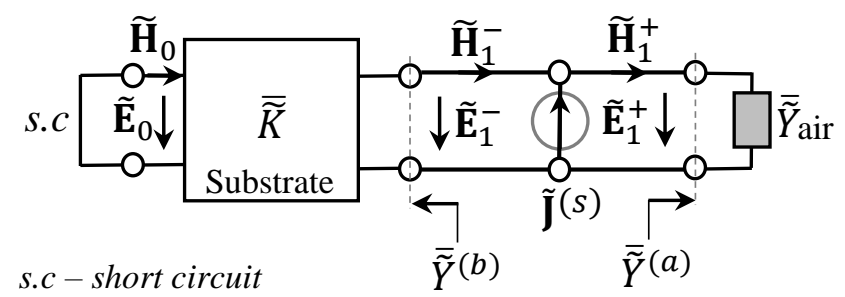

Fig. 2. Equivalent circuit model for the structure illustrated in the Fig. 1.

The matrix terms $\tilde{G}_{y y}, \tilde{G}_{y x}, \tilde{G}_{x y}$ and $\tilde{G}_{x x}$ of the Eq. 2 are given by

$$
\tilde{G}_{y y}=\frac{D\left[D \tilde{Y}_{2,2}^{(a)}+\tilde{B}_{2,2} \tilde{Z}_{1,1}\right]}{T_{\zeta}},
$$




$$
\begin{aligned}
\tilde{G}_{y x} & =\frac{D\left[\tilde{B}_{1,1} \tilde{Z}_{1,2}-D \tilde{Y}_{1,2}^{(a)}\right]}{T_{\zeta}}, \\
\tilde{G}_{x y} & =\frac{D\left[\tilde{B}_{2,2} \tilde{Z}_{2,1}-D \tilde{Y}_{2,1}^{(a)}\right]}{T_{\zeta}},
\end{aligned}
$$

and

$$
\tilde{G}_{x x}=\frac{D\left[D \tilde{Y}_{1,1}^{(a)}+\tilde{B}_{1,1} \tilde{Z}_{2,2}\right]}{T_{\zeta}}
$$

where

$$
\begin{gathered}
D=\operatorname{det}(\overline{\tilde{Z}})=\tilde{Z}_{1,1} \tilde{Z}_{2,2}-\tilde{Z}_{1,2} \tilde{Z}_{2,1} \\
T_{\zeta}=\left(D \tilde{Y}_{2,2}^{(a)}+\tilde{B}_{2,2} \tilde{Z}_{1,1}\right)\left(D \tilde{Y}_{1,1}^{(a)}+\tilde{B}_{1,1} \tilde{Z}_{2,2}\right)-\left(D \tilde{Y}_{1,2}^{(a)}-\tilde{B}_{1,1} \tilde{Z}_{1,2}\right)\left(D \tilde{Y}_{2,1}^{(a)}-\tilde{B}_{2,2} \tilde{Z}_{2,1}\right),
\end{gathered}
$$

and

$$
\overline{\tilde{\psi}}=\left[\begin{array}{cc}
\tilde{\psi}_{1,1} & \tilde{\psi}_{1,2} \\
\tilde{\psi}_{2,1} & \tilde{\psi}_{2,2}
\end{array}\right]
$$

with $\psi=B, Z$ or $Y^{(a)}$, and $\tilde{\psi}_{i, j}$ denote the matrix terms $i, j=1,2$.

The surface waves in the structure (modes) can be numerically determined by doing $T_{\zeta}=0$. An example of the propagation modes that can be computed with this formulation with the variation of the substrate thickness is presented in Fig. 3 for the first six modes.

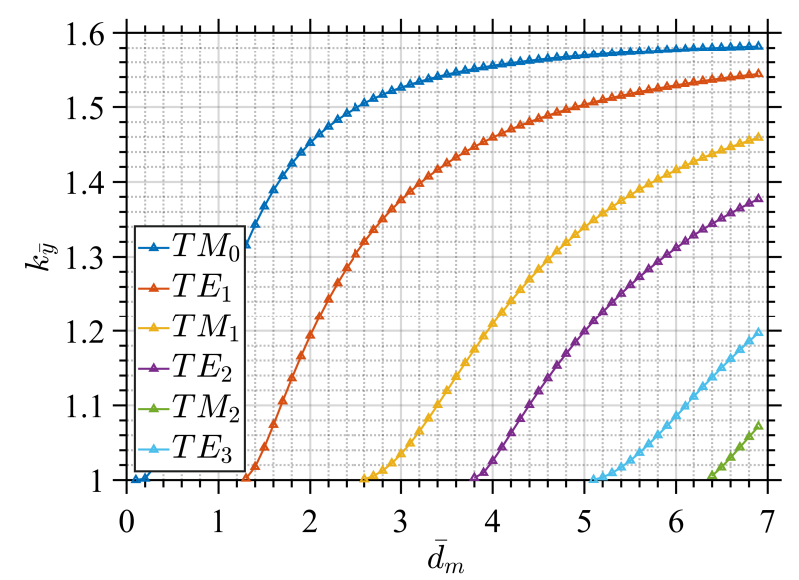

Fig. 3. Surface wave propagation constants for the grounded dielectric slab of Fig. 1, with $k_{y}=k_{\bar{y}} k_{0}, \bar{d}_{m}=d_{m} k_{0}$, and $\varepsilon_{r_{1}}=2.55$.

\section{B. Method of Moments - MoM}

The solution for the induced current applies the MoM approach to structures composed of $Q=Q_{y} Q_{x}$ printed elements (as illustrated in Fig. 4), where $Q_{y}$ and $Q_{x}$ are the number of scatterers along the $y$ and $x$ directions.

The resulting matrix equation is given by

$$
\bar{Z} \mathbf{I}=\mathbf{V}
$$

where $\mathbf{V}$ and $\mathbf{I}$ are the vectors with dimensions $\left(N_{y}+N_{x}\right) \times 1$ and $\bar{Z}$ is a matrix with $\left(N_{y}+N_{x}\right) \times$ $\left(N_{y}+N_{x}\right)$ elements, where $N_{y}$ and $N_{x}$ are the number of modes used to expand the current along the 


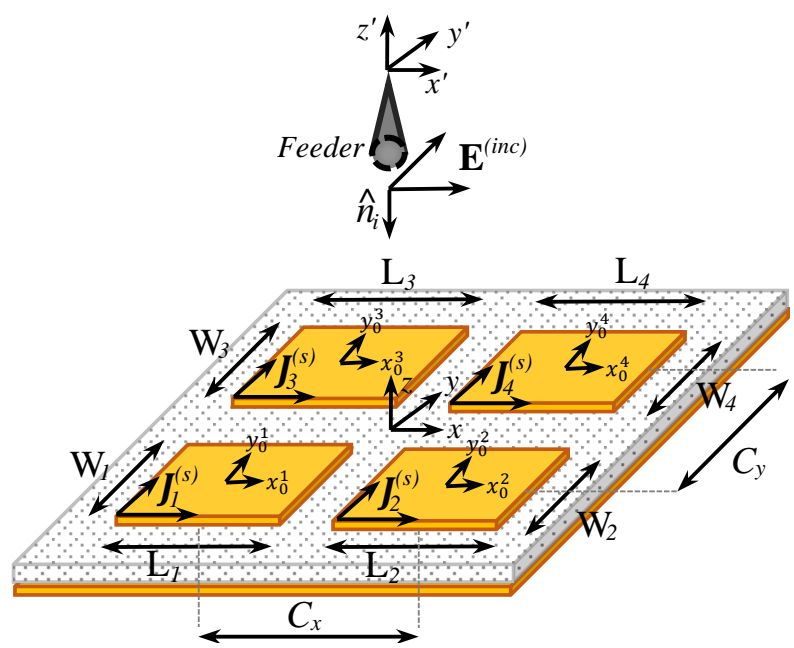

Fig. 4. Reflectarray antenna composed of four squared elements.

$y$ and $x$ directions. The impedance matrix is described by

$$
\begin{gathered}
\bar{Z}=\left[\begin{array}{ccccc}
\bar{Z}^{1,1} & \ldots & \bar{Z}^{1, q} & \ldots & \bar{Z}^{1, Q} \\
\bar{Z}^{p, 1} & \ldots & \bar{Z}^{p, q} & \ldots & \bar{Z}^{p, Q} \\
\bar{Z}^{Q, 1} & \ldots & \bar{Z}^{Q, q} & \ldots & \bar{Z}^{Q, Q}
\end{array}\right], \\
\bar{Z}^{p, q}=\left[\begin{array}{lll}
\bar{Z}^{y, y ; p, q} & \bar{Z}^{y, x ; p, q} \\
\bar{Z}^{x, y ; p, q} & \bar{Z}^{x, x ; p, q}
\end{array}\right], \\
\bar{Z}^{i, j ; p, q}=\left[\begin{array}{ccc}
Z_{1,1}^{i, j ; p, q} & Z_{1, n_{j}}^{i, j ; p, q} & Z_{1, N_{j}}^{i, j ; p, q} \\
Z_{n_{i}, 1,1, p}^{i, j} & Z_{n_{i}, n_{j}}^{i, j, p} & Z_{n_{i}, N_{j}}^{i, j, q} \\
Z_{N_{i}, 1}^{i, j ; p, q} & Z_{N_{i}, n_{j}}^{i, j p, q} & Z_{N_{i}, N_{j}}^{i, j, p}
\end{array}\right],
\end{gathered}
$$

where each term of the impedance matrix is determined using

$$
Z_{m, n}^{i, j ; p, q}=\int_{-\infty}^{+\infty} \int_{-\infty}^{+\infty}-\frac{j \eta_{0} \tilde{G}_{i j}}{(2 \pi)^{2}} \mathcal{F}\left(J_{m_{i}}^{p *}\right) \mathcal{F}\left(J_{n_{j}}^{q}\right) d k_{x} d k_{y},
$$

with $i, j=y, x$, and the indices $m$ and $n$ denote the expansion modes. The voltage vector is expressed by

$$
\begin{gathered}
\mathbf{V}=\left[\begin{array}{llll}
\mathbf{V}^{1} \ldots \mathbf{V}^{p} & \ldots \mathbf{V}^{Q}
\end{array}\right]^{t} \\
\mathbf{V}^{p}=\left[\begin{array}{lllll}
V_{1}^{y ; p} & \ldots V_{n_{y}}^{y ; p} \ldots V_{N_{y}}^{y ; p} & V_{1}^{x ; p} \ldots V_{n_{x}}^{x ; p} \ldots V_{N_{x}}^{x ; p}
\end{array}\right]^{t}
\end{gathered}
$$

where each term of the voltage vector is obtained from

$$
V_{m}^{i ; p}=\int_{y_{1}^{p}}^{y_{2}^{p}} \int_{x_{1}^{p}}^{x_{2}^{p}} E_{i}^{(i n c)}(x, y) J_{m_{i}}^{p} d x d y
$$

being $x_{1}^{p}=x_{o}^{p}-L_{p} / 2, x_{2}^{p}=x_{o}^{p}+L_{p} / 2, y_{1}^{p}=y_{o}^{p}-W_{p} / 2$ and $y_{2}^{p}=y_{o}^{p}+W_{p} / 2, x_{o}^{p}$ and $y_{o}^{p}$ are the centre coordinates of the $p$-th patch surface, $E_{i}^{(i n c)}$ is the incident electric field (plane wave), $\mathcal{F}\left(J_{n_{j}}^{q}\right)$ is the Fourier transform of the $n$-th basis function and $\mathcal{F}\left(J_{m_{i}}^{p *}\right)$ is the Fourier transform of the $m$-th 
complex-conjugated test function.

The tangential components of the electric field radiated by the feeder on the patches of the Fig. 4 can be described by

$$
\begin{aligned}
& E_{x}^{(i n c)}(x, y)=\left[E_{2_{\|}} \cos \theta_{i} \cos \phi_{i}+E_{2_{\perp}} \sin \phi_{i}\right] e^{j\left(k_{x} x+k_{y} y+k_{z_{2}} d_{m}\right)}, \\
& E_{y}^{(i n c)}(x, y)=\left[E_{2_{\|}} \cos \theta_{i} \sin \phi_{i}-E_{2_{\perp}} \cos \phi_{i}\right] e^{j\left(k_{x} x+k_{y} y+k_{z_{2}} d_{m}\right)},
\end{aligned}
$$

where $\phi_{i}$ and $\theta_{i}$ are the incidence coordinates, $E_{2_{\|}}$and $E_{2_{\perp}}$ are the amplitude of the parallel and perpendicular components of the electric field in region 2 (air).

In order to account for the steep increase of the current density near the non-radiating edges of the patches, the standard sinusoidal basis function [9] has been modified to assume the following form

$$
\begin{aligned}
& J_{n_{x}}^{q}(x, y)=J_{y}^{q}(y) \sin \left(\frac{n \pi}{L_{q}}\left(x-\left(x_{o}^{q}-L_{q} / 2\right)\right)\right), \\
& J_{n_{y}}^{q}(x, y)=J_{x}^{q}(x) \sin \left(\frac{n \pi}{W_{q}}\left(y-\left(y_{o}^{q}-W_{q} / 2\right)\right)\right),
\end{aligned}
$$

with

$$
J_{\xi}^{q}(\xi)=\left\{\begin{array}{cc}
\frac{1}{U_{\xi}}\left[1-\left(\frac{\xi-\xi_{01}}{\xi_{b}}\right)^{2}\right]^{-1 / 2}, & \xi_{1}<\xi \leq \xi_{01} \\
\frac{1}{U_{\xi}} & , \xi_{01}<\xi<\xi_{02} \\
\frac{1}{U_{\xi}}\left[1-\left(\frac{\xi-\xi_{02}}{\xi_{b}}\right)^{2}\right]^{-1 / 2}, \xi_{02} \leq \xi<\xi_{2} \\
\xi_{01}=\xi_{1}+\xi_{b}, \\
\xi_{02}=\xi_{2}-\xi_{b} \\
\xi_{b}=\kappa\left[\frac{\xi_{2}-\xi_{1}}{2}\right]
\end{array}\right.
$$

$\xi=x, y, U_{x}=L_{q}, U_{y}=W_{q}$, where $\xi_{1}$ and $\xi_{2}$ denote the positions of the $q$-th patch edges along the $\xi$-direction. The parameter $\kappa$ models the current density growth near to the patch edges parallel to the current flow and can assume any value in the interval $[0,1]$. Fig. 5 illustrates the isometric view of the surface electric current density for the first mode $(n=1)$ and $\kappa=0.4$.

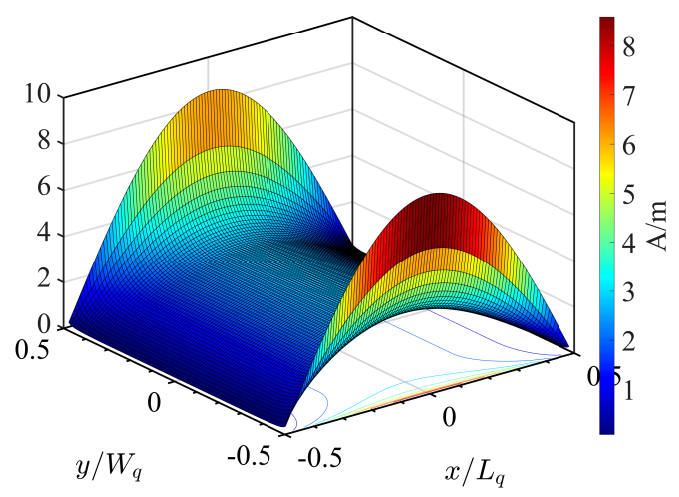

Fig. 5. Three-dimensional view of surface electric current density for $n=1$ and $\kappa=0.4$. 
Fig. 6 shows the behavior of the sinusoidal basis function for several modes and the influence of $\kappa$ parameter on the shape of the basis function along the $y$-direction.

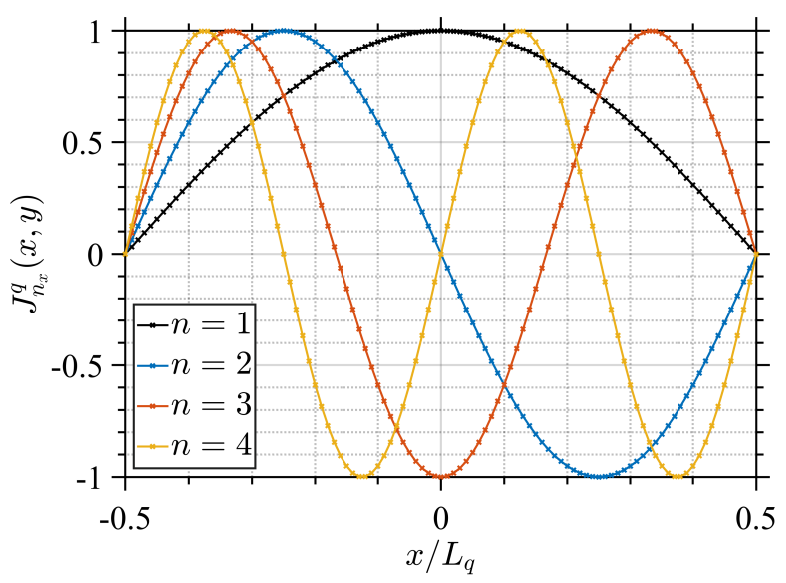

(a)

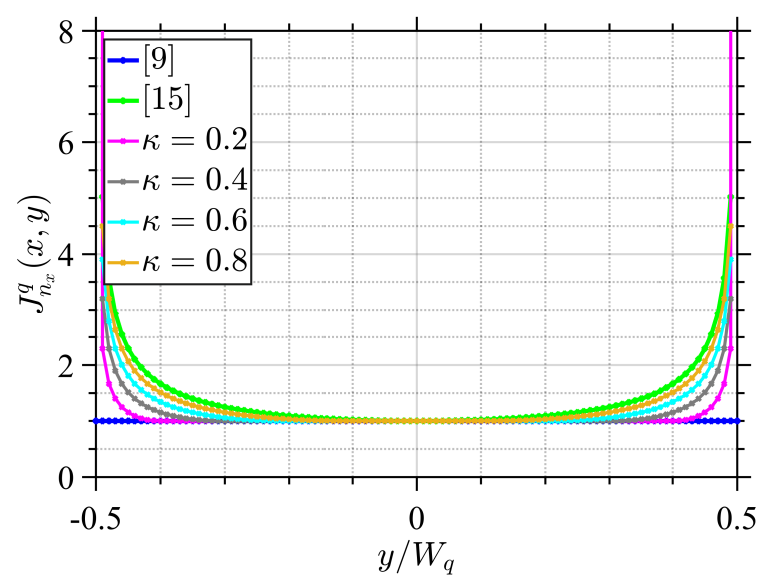

(b)

Fig. 6. Sinusoidal basis function with segmented edge condition. a) Modes used to expand the surface electric current $(y=0)$; b) Influence of $\kappa$ on the shape in the direction normal to the current flow $(x=0)$.

If $\kappa=0$, the formulation results in a simple sinusoidal function without edge condition [9]. If $\kappa=1$, then the condition is applied over the entire width of patch, resulting in the formulation with sinusoidal with continuous edge condition (without segmentation) [15].

The Fourier transforms of (25) and (26) are written as

$$
\begin{aligned}
& \tilde{J}_{n_{x}}^{q}\left(k_{x}, k_{y}\right)=\xi_{y}^{q}\left(k_{y}, W_{q}, \kappa\right) \frac{n \pi}{L_{q}}\left[\frac{\cos (n \pi) e^{-j \frac{1}{2} k_{x} L_{q}}-e^{j \frac{1}{2} k_{x} L_{q}}}{k_{x}^{2}-\left(\frac{n \pi}{L_{q}}\right)^{2}}\right] \zeta_{q}^{\prime}, \\
& \tilde{J}_{n_{y}}^{q}\left(k_{x}, k_{y}\right)=\xi_{x}^{q}\left(k_{x}, L_{q}, \kappa\right) \frac{n \pi}{W_{q}}\left[\frac{\cos (n \pi) e^{-j \frac{1}{2} k_{y} W_{q}}-e^{j \frac{1}{2} k_{y} W_{q}}}{k_{y}^{2}-\left(\frac{n \pi}{W_{q}}\right)^{2}}\right] \zeta_{q}^{\prime},
\end{aligned}
$$


with

$$
\begin{aligned}
& \xi_{y}^{q}\left(k_{y}, W_{q}, \kappa\right)=(1-\kappa) \operatorname{sinc}\left(\frac{W_{q} k_{y}}{2}(-1+\kappa)\right) \\
&+\frac{\pi \kappa}{2}[ J_{0}\left(\frac{\kappa W_{q} k_{y}}{2}\right) \cos \left(\frac{W_{q} k_{y}}{2}(-1+\kappa)\right) \\
&\left.-j \mathcal{L}_{0}\left(\frac{\kappa W_{q} k_{y}}{2}\right) \sin \left(\frac{W_{q} k_{y}}{2}(-1+\kappa)\right)\right], \\
& \xi_{x}^{q}\left(k_{x}, L_{q}, \kappa\right)=(1-\kappa) \operatorname{sinc}\left(\frac{L_{q} k_{x}}{2}(-1+\kappa)\right) \\
&+\frac{\pi \kappa}{2}\left[J_{0}\left(\frac{\kappa L_{q} k_{x}}{2}\right) \cos \left(\frac{L_{q} k_{x}}{2}(-1+\kappa)\right)\right. \\
&\left.-j \mathcal{L}_{0}\left(\frac{\kappa L_{q} k_{x}}{2}\right) \sin \left(\frac{L_{q} k_{x}}{2}(-1+\kappa)\right)\right],
\end{aligned}
$$

and

$$
\zeta_{q}^{\prime}=e^{-j\left(k_{x} x_{o}^{q}+k_{y} y_{o}^{q}\right)}
$$

where $x_{o}^{q}$ and $y_{o}^{q}$ are the centre coordinates of the $q$-th patch surface, $J_{0}$ is the Bessel function of the first kind and order zero, and $\mathcal{L}_{0}$ is the modified Struve function of order zero, which can be evaluated as

$$
\mathcal{L}_{0}(\gamma)=\frac{j 2}{\pi} \int_{0}^{\frac{\pi}{2}} \sin [\gamma \cos (v)] \partial v .
$$

The Fourier transform of the complex-conjugated test function is given by

$$
\begin{aligned}
& \tilde{J}_{m_{x}}^{p *}\left(k_{x}, k_{y}\right)=\xi_{y}^{p}\left(k_{y}, W_{p}, \kappa\right) \frac{m \pi}{L_{q}}\left[\frac{\cos (m \pi) e^{j \frac{1}{2} k_{x} L_{p}}-e^{-j \frac{1}{2} k_{x} L_{p}}}{k_{x}^{2}-\left(\frac{m \pi}{L_{p}}\right)^{2}}\right] \zeta_{q}^{*}, \\
& \tilde{J}_{m_{y} *}^{p *}\left(k_{x}, k_{y}\right)=\xi_{x}^{p}\left(k_{x}, L_{p}, \kappa\right) \frac{m \pi}{W_{p}}\left[\frac{\cos (m \pi) e^{j \frac{1}{2} k_{y} W_{p}}-e^{-j \frac{1}{2} k_{y} W_{p}}}{k_{y}^{2}-\left(\frac{m \pi}{W_{p}}\right)^{2}}\right] \zeta_{q}^{*},
\end{aligned}
$$

with

$$
\begin{aligned}
\xi_{y}^{p}\left(k_{y}, W_{p}, \kappa\right)=(1-\kappa) & \operatorname{sinc}\left(\frac{W_{p} k_{y}}{2}(-1+\kappa)\right) \\
+\frac{\pi \kappa}{2} & {\left[J_{0}\left(\frac{\kappa W_{p} k_{y}}{2}\right) \cos \left(\frac{W_{p} k_{y}}{2}(-1+\kappa)\right)\right.} \\
& \left.-j \mathcal{L}_{0}\left(\frac{\kappa W_{p} k_{y}}{2}\right) \sin \left(\frac{W_{p} k_{y}}{2}(-1+\kappa)\right)\right],
\end{aligned}
$$




$$
\begin{aligned}
\xi_{x}^{p}\left(k_{x}, L_{p}, \kappa\right)=(1-\kappa) & \operatorname{sinc}\left(\frac{L_{p} k_{x}}{2}(-1+\kappa)\right) \\
+\frac{\pi \kappa}{2} & {\left[J_{0}\left(\frac{\kappa L_{p} k_{x}}{2}\right) \cos \left(\frac{L_{p} k_{x}}{2}(-1+\kappa)\right)\right.} \\
& \left.-j \mathcal{L}_{0}\left(\frac{\kappa L_{p} k_{x}}{2}\right) \sin \left(\frac{L_{p} k_{x}}{2}(-1+\kappa)\right)\right],
\end{aligned}
$$

and

$$
\zeta_{p}^{\prime *}=e^{j\left(k_{x} x_{o}^{p}+k_{y} y_{o}^{p}\right)}
$$

The radiated electric field by a reflectarray antenna composed of $Q$ elements can be determined in the upper hemisphere $(z>0)$ using the asymptotic evaluation of integrals with stationary phase approximation [18], described here as

$$
\mathbf{E}(r, \theta, \phi)=j k_{0} \frac{e^{-j k_{0} r}}{2 \pi r} \sum_{q=1}^{Q} \hat{\theta} E_{\theta}^{q(s)}(\theta, \phi)+\hat{\phi} E_{\phi}^{q(s)}(\theta, \phi),
$$

where

$$
\begin{gathered}
E_{\theta}^{q(s)}(\theta, \phi)=\tilde{E}_{x}^{q(s)}\left(k_{x}, k_{y}\right) \cos \phi+\tilde{E}_{y}^{q(s)}\left(k_{x}, k_{y}\right) \sin \phi \\
E_{\phi}^{q(s)}(\theta, \phi)=\left[-\tilde{E}_{x}^{q(s)}\left(k_{x}, k_{y}\right) \sin \phi+\tilde{E}_{y}^{q(s)}\left(k_{x}, k_{y}\right) \cos \phi\right] \cos \theta,
\end{gathered}
$$

with $k_{x}=k_{0} \sin \theta \cos \phi$, and $k_{y}=k_{0} \sin \theta \sin \phi$.

\section{NUMERICAL RESULTS}

To verify the performance of the proposed basis function, we consider the incidence of a plane wave operating at $2.4 \mathrm{GHz}$. The substrate relative permittivity is $\varepsilon_{r_{b}}=3.38$ and thickness is $d_{m}=1.524 \mathrm{~mm}$, which corresponds approximately to 0.012 wavelengths.

The MoM is implemented with Galerkin approach to obtain the coefficients (values of the $\mathbf{I}$ vector elements) for each mode used to expand the current density. For this study, only the first mode in each direction is considered, and, for comparison, the following entire-domain basis functions are considered: sinusoidal [9], sinusoidal with continuous edge condition [15], and sinusoidal with segmented edge condition (proposed in this work with $0.2 \leq \kappa \leq 0.8$ ). By considering a square element $(W=L)$ and varying the dimension $L$ (see Fig. 1), Fig. 7 shows the results for the phase and the magnitude of the scattered electric field, along with the results provided by the electromagnetic simulator Ansys Designer, which is a well-known commercial simulation package based on MoM with subdomain basis functions and is used for validation of the segmented edge condition $(\kappa)$. The simulations with Ansys Designer were carried out using Planar EM Solver by setting the solution frequency to $2.4 \mathrm{GHz}$. The use of edge mesh option was set with length ratio equal to 0.1 .

Only very small discrepancies can be verified between the results of the proposed approach with $\kappa=0.35$ and the values obtained with the commercial software. The maximum errors are $\pm 5^{\circ}$ for the phase and $0.8 \mathrm{~dB}$ for the amplitude. Figure 7 shows that the maximum scattering occurs for the dimension in which the structure resonates. For thin substrates, the resonance size can be estimated as [17]

$$
L=\frac{c}{2 f_{r} \sqrt{\varepsilon_{r_{b}}}},
$$

Brazilian Microwave and Optoelectronics Society-SBMO Brazilian Society of Electromagnetism-SBMag received 30 Jan 2021; for review 18 Feb 2021; accepted 28 June 2021 (C) 2021 SBMO/SBMag (cc) BY

ISSN 2179-1074 
where $c$ is the speed of light in free space and $f_{r}$ is the resonance frequency. The design of RAs with rectangular elements requires the application of thin substrates to increase the range of phase variation. Thus, the value of $\kappa$ parameter can be determined using an iterative process based on (45) and the ressonant behaviour. In the other words, the proposed procedure is carried out as follows: initially, the dimensions of the square scatterer are calculated using (45). Magnitude responses are calculated for several $\kappa$ values with our approach. The choice of $\kappa$ is done based on the calculated curves that produces the closest maximum amplitude to the calculated dimensions in comparison to the results of Ansys Designer. In Fig. 7, this is verified with the red curve, which corresponds to $\kappa=0.35$.

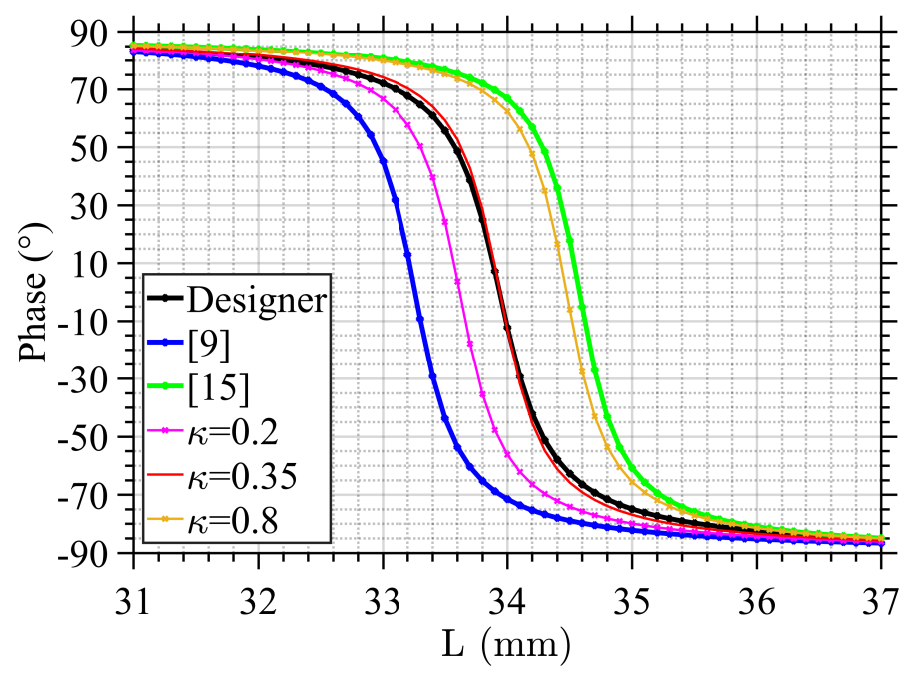

(a)

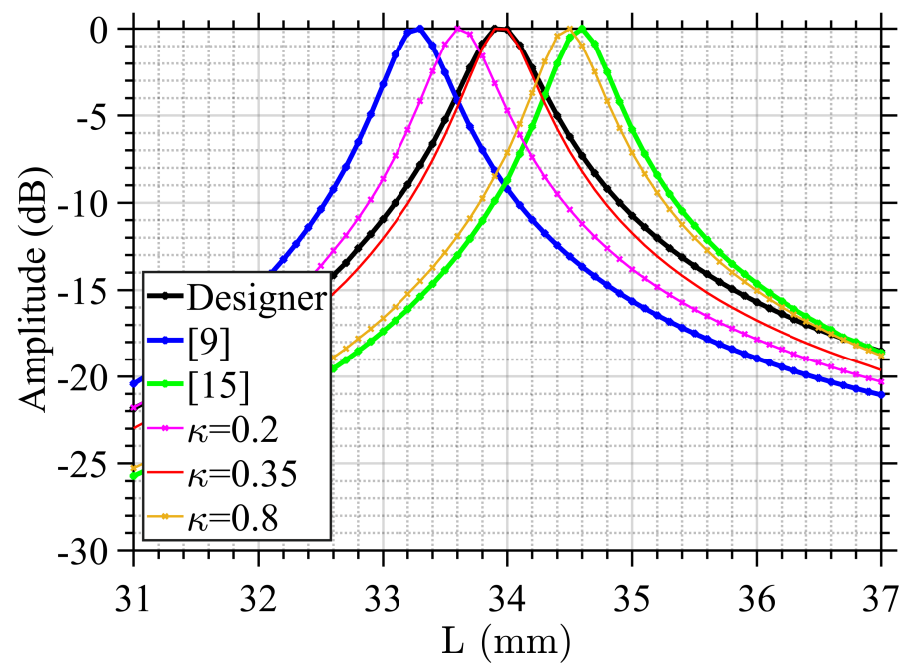

(b)

Fig. 7. Results for the scattered electric field by a rectangular patch. a) Phase curves; b) Magnitude curves.

Figure 8 displays the radiation pattern of a single element in the $\mathrm{E}$ and $\mathrm{H}$ planes for $\kappa=0.35$, where excellent agreement between the proposed approach and the commercial software can be verified.

To further demonstrate the performance of the basis function with segmented edge condition, the fields scattered by $3 \times 3$ and $5 \times 5$ reflectarrays are recalculated. These RAs are illuminated by a wave plane under normal and oblique incidences, and the spacing between the printed elements is $C_{y}=C_{x}=\lambda_{0} / 2$. 


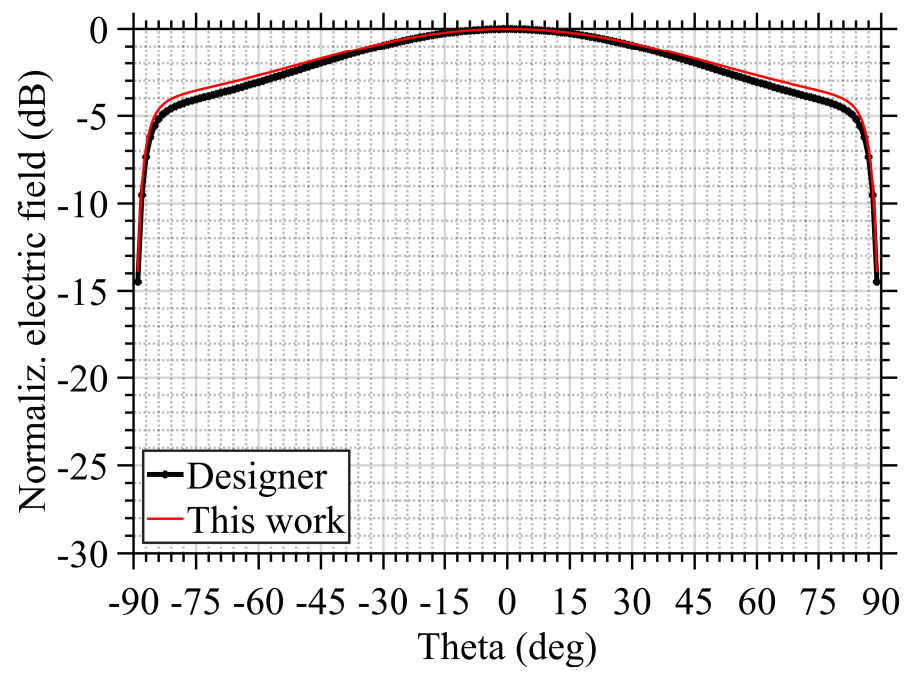

(a)

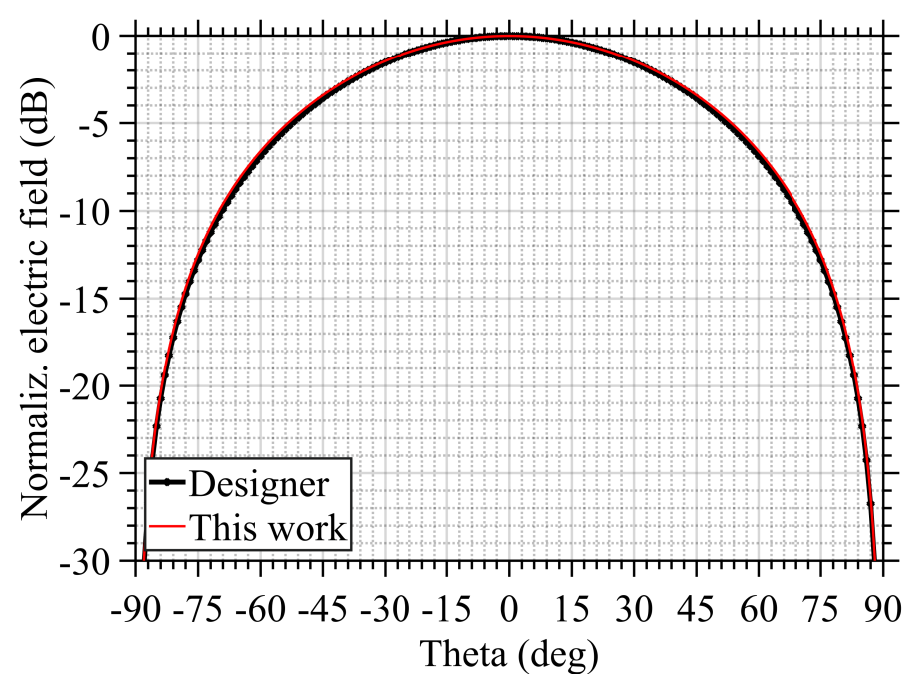

(b)

Fig. 8. Single element: calculated and simulated radiation patterns for $\kappa=0.35$. a) E-plane $E_{\theta}\left(\theta, \phi=0^{\circ}\right)$; b) H-plane $E_{\phi}\left(\theta, \phi=90^{\circ}\right)$.

Excellent agreement between the proposed approach and Ansys Designer can also be verified in Figs. 9, 10,11 and 12.

For these computations, Ansys Designer needed 10,935 and 14,942 unknowns for the $3 \times 3$ and $5 \times 5$ RAs, respectively, whereas the proposed formulation demanded only 18 and 50 unknowns. 


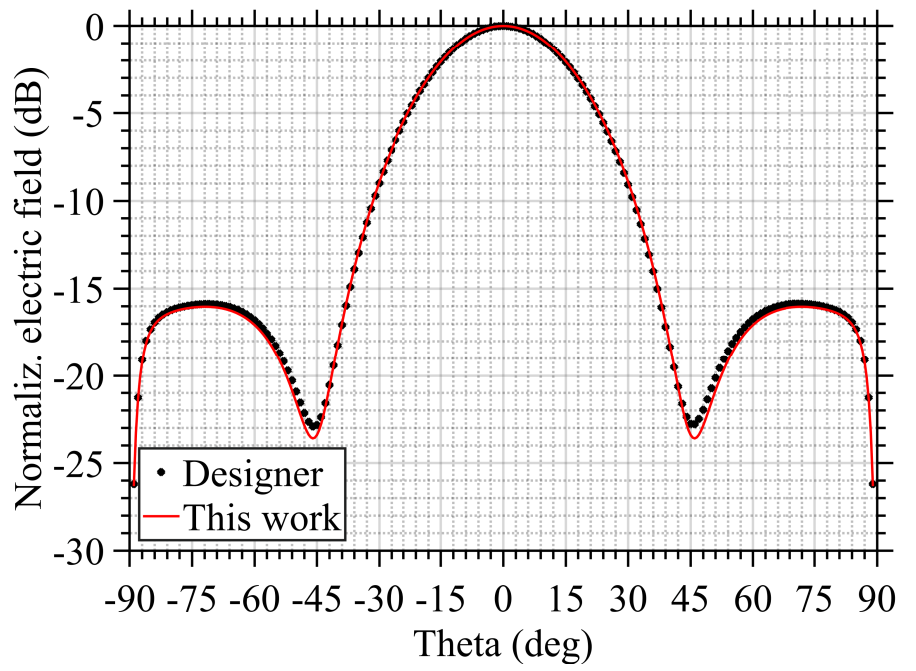

(a)

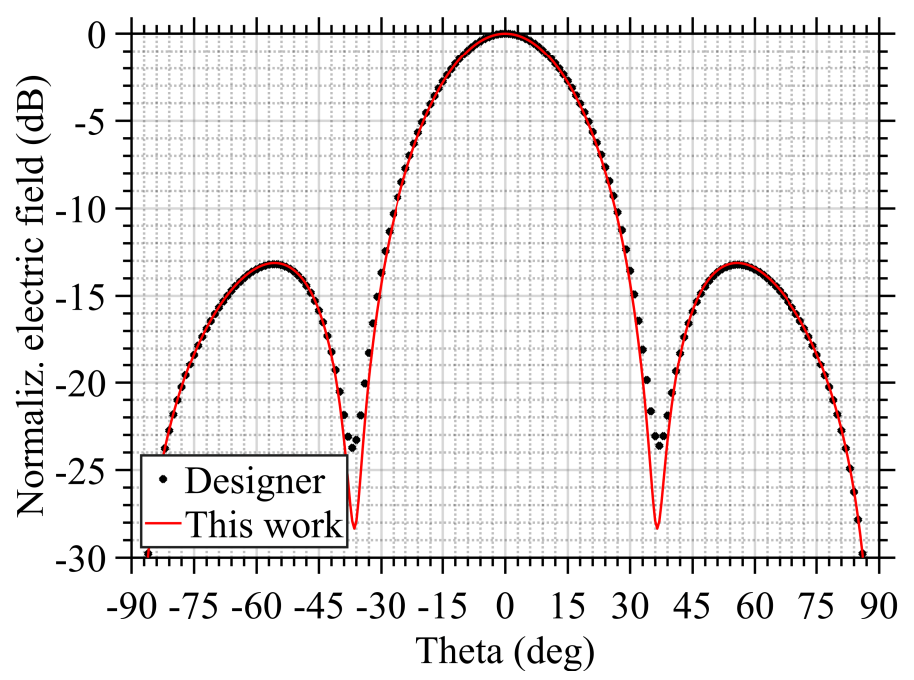

(b)

Fig. 9. RA $3 \times 3$ : calculated and simulated radiation patterns for $\kappa=0.35$. Normal incidence: a) E-plane $E_{\theta}\left(\theta, \phi=0^{\circ}\right)$; b) H-plane $E_{\phi}\left(\theta, \phi=90^{\circ}\right)$. 


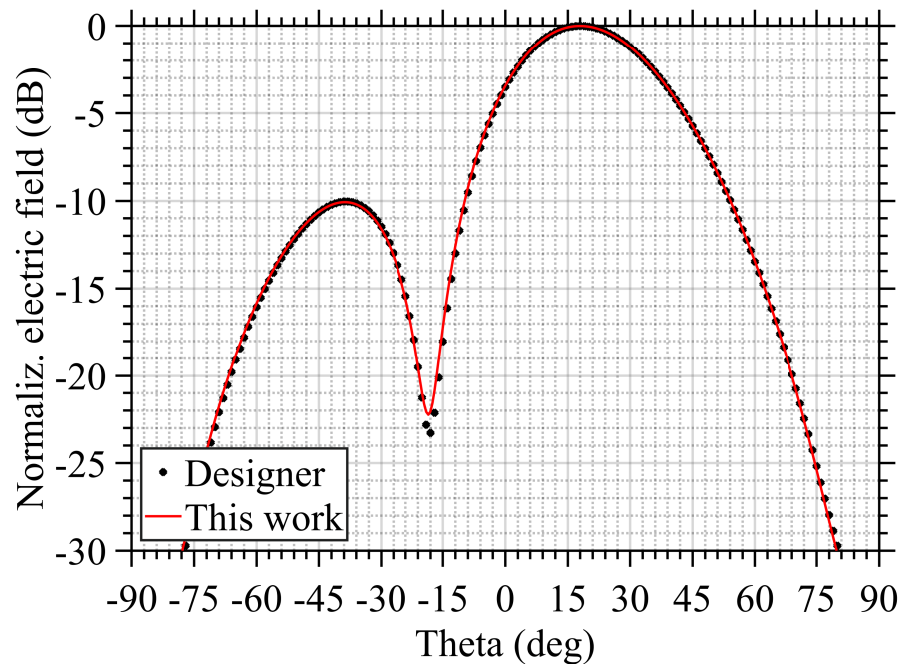

(a)

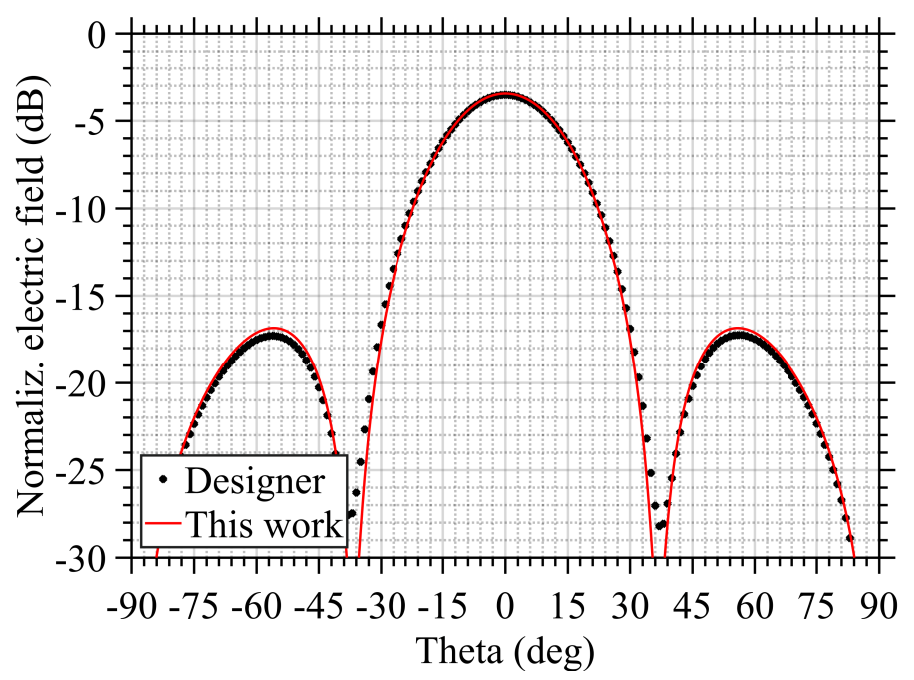

(b)

Fig. 10. RA $3 \times 3$ : calculated and simulated radiation patterns for $\kappa=0.35$. Oblique incidence $\left(\theta_{i}=20^{\circ}\right)$ : a) E-plane $E_{\theta}\left(\theta, \phi=0^{\circ}\right)$; b) $E_{\phi}\left(\theta, \phi=90^{\circ}\right)$. 


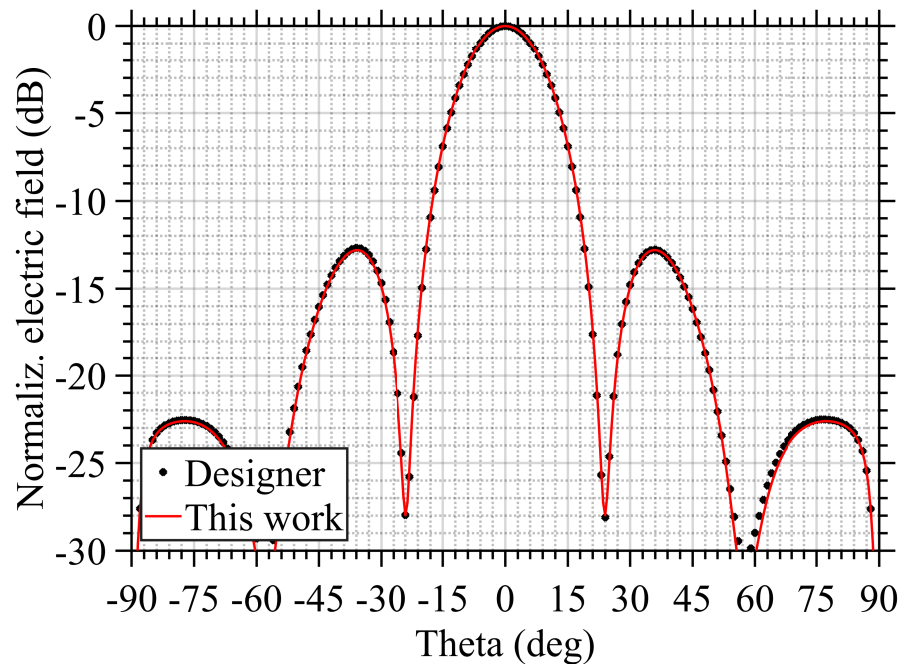

(a)

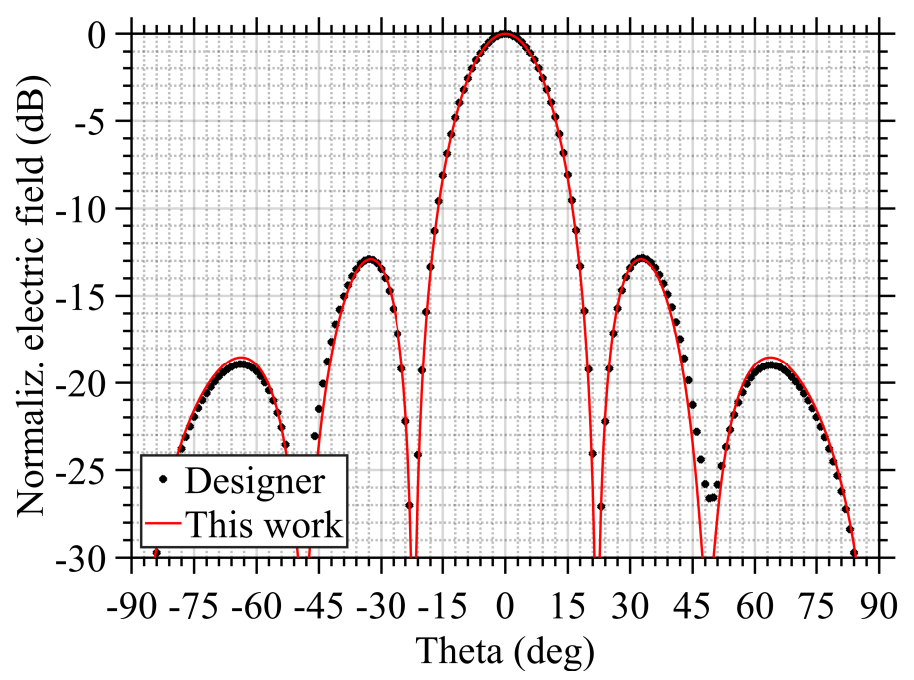

(b)

Fig. 11. RA $5 \times 5$ : calculated and simulated radiation patterns for $\kappa=0.35$. Normal incidence: a) E-plane $E_{\theta}\left(\theta, \phi=0^{\circ}\right)$; b) H-plane $E_{\phi}\left(\theta, \phi=90^{\circ}\right)$. 


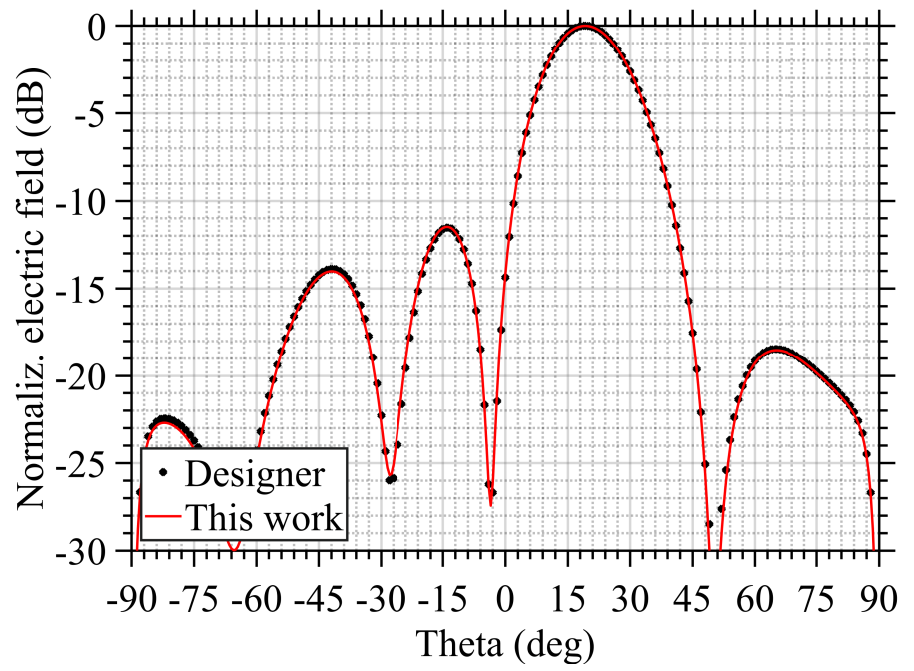

(a)

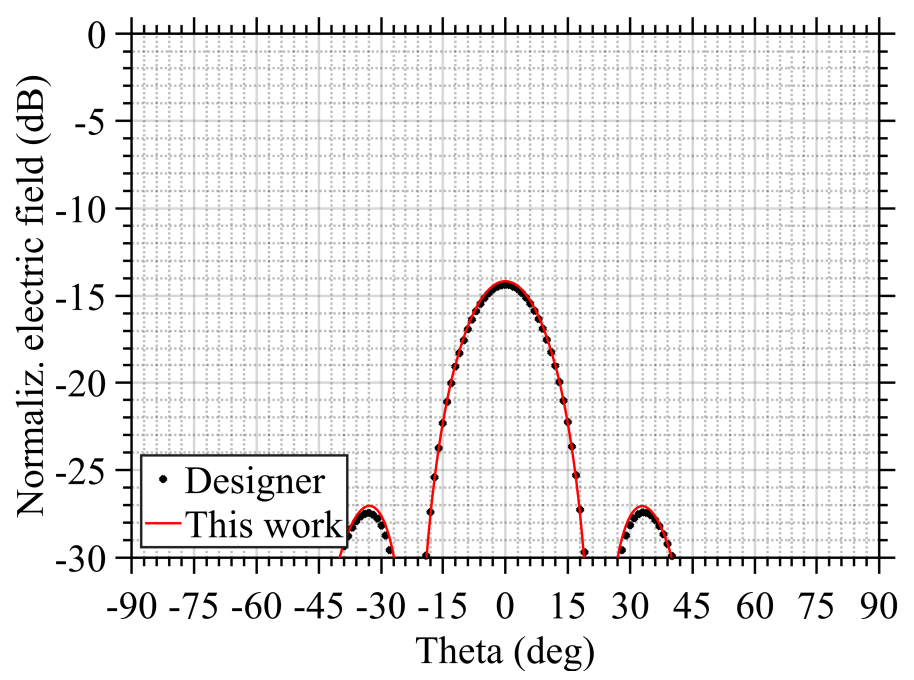

(b)

Fig. 12. RA $5 \times 5$ : calculated and simulated radiation patterns for $\kappa=0.35$. Oblique incidence $\left(\theta_{i}=20^{\circ}\right)$ : a) E-plane $E_{\theta}\left(\theta, \phi=0^{\circ}\right)$; b) $E_{\phi}\left(\theta, \phi=90^{\circ}\right)$. 


\section{CONCLUSION}

The application of an entire-domain basis function with segmented edge condition has been presented for the computation of the scattered field of rectangular microstrip patches. By performing numerical analyses, this basis function demonstrated to be very efficient, since only two modes were necessary to model rectangular patch scatterers accurately. The results obtained have been validated by comparison to radiation pattern calculated with Ansys Designer, whereby the proposed approach used less than $1 \%$ of the unknowns demanded by the commercial software. This is an interesting feature for the analysis of large reflectarrays.

\section{REFERENCES}

[1] E. Almajali, D. A. McNamara, J. Shaker, and M. R. Chaharmir, "Feed image lobes in offset-fed reflectarrays: Diagnosis and solution," IEEE Transactions on Antennas and Propagation, vol. 62, no. 1, pp. 216-227, 2014.

[2] P. Nayeri, A. Z. Elsherbeni, and F. Yang, "Radiation analysis approaches for reflectarray antennas [antenna designer's notebook]," IEEE Antennas and Propagation Magazine, vol. 55, no. 1, pp. 127-134, 2013.

[3] M. Karimipour, A. Pirhadi, and N. Ebrahimi, "Accurate method for synthesis of shaped-beam non-uniform reflectarray antenna," IET Microwaves, Antennas Propagation, vol. 7, no. 15, pp. 1247-1253, 2013.

[4] D. R. Prado, M. Arrebola, M. R. Pino, R. Florencio, R. R. Boix, J. A. Encinar, and F. Las-Heras, "Efficient crosspolar optimization of shaped-beam dual-polarized reflectarrays using full-wave analysis for the antenna element characterization," IEEE Transactions on Antennas and Propagation, vol. 65, no. 2, pp. 623-635, 2017.

[5] I. Bardi, R. Remski, D. Perry, and Z. Cendes, "Plane wave scattering from frequency-selective surfaces by the finiteelement method," IEEE Transactions on Magnetics, vol. 38, no. 2, pp. 641-644, 2002.

[6] J. Huang and J. A. Encinar, Antenna Theory: Analysis and Design. Wiley, New Jersey, 2005.

[7] C. A. Balanis, Reflectarray antennas. Wiley, New York, 2008.

[8] L. Gürel, K. Sertel, and K. Şendur, "On the choice of basis functions to model surface electric current densities in computational electromagnetics," Radio Science, vol. 34, no. 6, pp. 1373-1387, 1999.

[9] D. Pozar, "Input impedance and mutual coupling of rectangular microstrip antennas," IEEE Transactions on Antennas and Propagation, vol. 30, no. 6, pp. 1191-1196, 1982.

[10] N. Aouabdia, F. Benabdelaziz, F. Bouttout, C. Zebiri, and F. Benmeddour, "Resonant frequency of a rectangular microstrip antenna depends of the dielectric substrate parameters using the various types of current expansion function," in First International Symposium on Control, Communications and Signal Processing, 2004., pp. 347-350, 2004.

[11] R. Florencio, R. R. Boix, E. Carrasco, J. A. Encinar, and V. Losada, "Efficient numerical tool for the analysis and design of reflectarrays based on cells with three parallel dipoles," Microwave and Optical Technology Letters, vol. 55, no. 6, pp. 1212-1216, 2013.

[12] T. Itoh, Numerical techniques for microwave and millimeter-wave passive structures. Wiley, New York, 1989.

[13] M. Zhou, E. Jorgensen, O. S. Kim, S. B. Sorensen, P. Meincke, and O. Breinbjerg, "Accurate and efficient analysis of printed reflectarrays with arbitrary elements using higher-order hierarchical legendre basis functions," IEEE Antennas and Wireless Propagation Letters, vol. 11, pp. 814-817, 2012.

[14] R. Florencio, R. R. Boix, and J. A. Encinar, "Fast and accurate mom analysis of periodic arrays of multilayered stacked rectangular patches with application to the design of reflectarray antennas," IEEE Transactions on Antennas and Propagation, vol. 63, no. 6, pp. 2558-2571, 2015.

[15] S. R. Rengarajan, "Choice of basis functions for accurate characterization of infinite array of microstrip reflectarray elements," IEEE Antennas and Wireless Propagation Letters, vol. 4, pp. 47-50, 2005.

[16] A. Dreher, "A new approach to dyadic green's function in spectral domain," IEEE Transactions on Antennas and Propagation, vol. 43, no. 11, pp. 1297-1302, 1995.

[17] P. Bhartia, I. Bahl, R. Garg, and A. Ittipiboon, Microstrip Antenna Design Handbook. Artech House, Norwood, MA, USA, 2000.

[18] M. Zhou, S. B. Sorensen, E. Jorgensen, P. Meincke, O. S. Kim, and O. Breinbjerg, "An accurate technique for calculation of radiation from printed reflectarrays," IEEE Antennas and Wireless Propagation Letters, vol. 10, pp. 1081-1084, 2011. 